\title{
La industria cerámica europea en el siglo XXI. Retos tecnológicos y desafíos de la próxima década
}

\author{
J.ALBORS, J. L. HERVÁS \\ Departamento de Organización de Empresas. Universidad Politécnica de Valencia
}

\begin{abstract}
La industria cerámica europea, española e italiana, ha logrado un liderazgo tecnológico importante en la actualidad. Sin embargo, en algunos aspectos del proceso de fabricación de la cerámica, subsisten culturas artesanales que aún perduran y que son derivadas del origen de la industria. Esta situación, combinada con la crisis iniciada en la actualidad, la globalización, la creciente presión de industrias cerámicas tan alejadas como la China y los cambios en las preferencias de los clientes presentan un panorama que va a acabar produciendo, previsiblemente, ciertas demandas tecnológicas sobre el proceso de manufactura cerámica, tras una fase previa de presión sobre el proceso de desarrollo de producto.

La presente comunicación tratara de realizar una reflexión sobre estos aspectos desde la perspectiva de los trabajos desarrollados por el proyecto MONOTONE, financiado por la Comisión Europea y en el que participan 17 institutos y empresas europeas del sector ${ }^{1}$.
\end{abstract}

Palabras Clave. Tecnología, Demanda, Cambio, Globalización industria cerámica

The European tile ceramic industry in the XXI century. Challenges of the present decade.

The European, Spanish and Italian tile ceramic industry, has achieved today a relevant technology leadership. Nevertheless, in certain aspects of the tile ceramic manufacturing process, artisan cultures still subsist which are derived from the origin of the industry. This situation, combined with the crisis initiated at the start of the century, the globalisation, the increasing pressure of tile ceramic industries located in the East and the changes in the preferences of the customers presents/ a scenario which will cause determined technology demands on the tile ceramic process.

The present communication reflects on these aspects from the perspective of the work developed by the MONOTONE project, financed by the European Commission.

Key words. Technology Demand, Change, Globalization

\section{INTRODUCCIÓN}

El sector industrial cerámico mundial presenta la peculiaridad de que se organiza, principalmente, en torno a unas concentraciones territoriales donde se localizan la mayoría de los agentes o industrias participantes en el sistema de creación de valor del producto final cerámico, tanto los fabricantes cerámicos como la industria proveedora de esmaltes y fritas y maquinaria cerámica, así como diversas instituciones públicas y privadas que apoyan y contribuyen al desarrollo de la industria. El desarrollo de estos "clusters" cerámicos ha propiciado que el sector en el ámbito mundial, aunque catalogado por la OCDE como de tecnología mediabaja, presente un grado de avance e innovación tecnológica punta (1) (2).

Un "cluster" regional (3) es un grupo de empresas localizadas en una proximidad geográfica, esto es, una aglomeración geográfica de empresas en sectores iguales o relacionados. A su vez, los distritos industriales, como los italianos (4)(5)(6)(7)(8) son concentraciones de empresas implicadas en un proceso de producción interdependiente, a menudo en la misma industria o segmento, que están arraigadas a la comunidad local y delimitadas por la distancia diaria al trabajo (9). Así, constatamos que los principales "clusters" cerámicos se localizan en Brasil, uno en Santa
Catarina y dos en el estado de Sao Paulo; en Portugal, en la zona de Aveiro; en Castellón, España; y finalmente, en la provincia de la Emilia Romagna, en Italia. Sin embargo, desde hace unos años, la contribución a la producción mundial de estas concentraciones territoriales apenas representa el 30\% aproximadamente. La razón reside en el nuevo protagonismo de la industria cerámica China que ostenta actualmente el 33\% (3) de la producción mundial. Este país ha crecido gracias a la tecnología de fritas y esmaltes desarrollada en el ámbito de Castellón y el sector de la maquinaria italiano, alcanzando con datos del 2002, no sólo el liderazgo mundial de producción, sino el tercer puesto con un 9,2\% en la cuota mundial de exportaciones, por detrás de España e Italia (3).

Ante este panorama dual, donde el sector de esmaltes y fritas y el de maquinaria cerámica se han expandido y potenciado con la entrada de China, aun sufriendo los últimos problemas derivados de la propiedad intelectual, el sector de fabricantes cerámicos empieza a afrontar un difícil camino donde se cuestionan diversas prácticas de funcionamiento, entre ellas las relacionadas con la escasa comercialización (marcas, estudios de mercado, canales de ventas, etc.) del producto cerámico, que aunque muy avanzado y con un gran valor añadido, no se logra transmitir al cliente final (2). Ante 
este nuevo panorama el objetivo del presente trabajo es doble. En primer lugar, se analizará y desarrollará la situación en la que actualmente se encuentra la industria cerámica en el mundo y, en especial, la europea para determinar el estado de la cuestión y establecer líneas futuras de actuación. Asimismo, en segundo lugar, analizaremos el impacto de la evolución del mercado cerámico mundial sobre la demanda tecnológica del sector.

Para la consecución de los objetivos presentados, el artículo se organizará como sigue. Tras esta introducción, el segundo punto se dedicará a la producción, demanda y comercio mundial. A continuación, se evaluará la industria cerámica y sus principales productores europeos. Seguidamente se abordará lo que ocurre en la industria cerámica europea desde el punto de vista de la demanda tecnológica. Por último, se establecerán las conclusiones y las líneas posibles de solución.

\section{DEMANDA Y COMERCIO MUNDIAL DEL AZULEJO CERÁMICO.}

\section{1.- La producción en el ámbito global: los nuevos países emergentes.}

La producción mundial sigue creciendo un 7,1\%, mientras que la producción europea decrece un 1,4\%. España, a pesar de la desaceleración europea, logra crecer al $2 \%$ en el año 2002. Así, China encabeza la producción mundial de azulejo con 2.240 millones de $\mathrm{m}^{2}$ para el año 2002 (3), produciendo casi el doble que Italia y España juntas. De esta manera, Italia y España pierden el liderazgo mundial de producción, si bien el producto Chino se destina en la mayoría de los casos al consumo interno y presenta aspectos de calidad que están a mucha distancia de los productores europeos. Tras España e Italia, con 651 y 605 millones de $\mathrm{m}^{2}$ respectivamente en el año 2002, se sitúan Brasil, 486 millones de $\mathrm{m}^{2}$, Turquía con 167 millones de $\mathrm{m}^{2}$, México con 151, e India con 137 millones de $\mathrm{m}^{2}$. La distribución de la cuota a nivel mundial queda así con un 36,3\% para China, el 9,5\% para Italia y 10,8 para España, el $7,9 \%$ para Brasil y en torno a un $2 \%$ para Turquía, para México y para la India. En lo que respecta a las variaciones anuales, aparte de los incrementos positivos de China, que en el año 2002 tuvo un 6,7\%, indicando cierta desaceleración, ya que en el año 2001 tuvo un crecimiento del 16,7\%, destacan Turquía con el 7,7\% y la India con el 16,9\% como más dinámicas que España con el $2 \%$ e Italia con el $-5,2 \%$ que retrocede de forma significativa (3).

\section{2.- El consumo mundial cerámico}

El principal país consumidor de azulejos, en términos absolutos, en el ámbito mundial es China, llegando a alcanzar un consumo de 2.117 millones de $\mathrm{m}^{2}$ en el año 2002, si bien su consumo per capita es relativamente bajo con 1,6 metros cuadrados / habitante. A su vez, España es, con diferencia, el mayor consumidor per cápita del mundo con $8,2 \mathrm{~m}^{2}$ por habitante, seguido por Portugal con $6,9 \mathrm{~m}^{2}$ por habitante, si bien con 327 millones de $\mathrm{m}^{2}$ totales España supera ampliamente a Portugal con 69 millones de m. ${ }^{2}$ (3)

En cuanto a consumo total en cifras absolutas de $\mathrm{m}^{2}$ (3), España queda en tercer lugar por detrás los de China con 2.117 millones de m. $^{2}$ y Brasil con 413 millones de m. $^{2}$, si bien estos dos últimos países, aunque tengan altos consumos absolutos, presentan consumos per cápita muy bajos, China con 1,6 m. ${ }^{2} /$ habitante y Brasil con 2,3 m. ${ }^{2} /$ habitante. A continuación, en términos de consumo absoluto siguen EE.UU., Italia, Alemania, México y Francia. Asimismo, España, con 8,2 m²/ habitante, presenta una gran diferencia con respecto a Italia con $3,2 \mathrm{~m}^{2} /$ habitantes en consumo per cápita.

Asimismo, observamos como, en el ámbito mundial, el consumo aumenta un $7,6 \%$, mientras que en la UE disminuye en un $0,4 \%$. Evidentemente, hay que pensar que la disminución del consumo europeo se debe, en parte, a las bajadas de los mercados más importantes como Alemania con 12,9\% y Francia con 1,3\%) e incluso Italia con $4,2 \%$.

\section{3.- El comercio mundial cerámico.}

Italia supone la mitad del total de exportación mundial, mientras que España tiene, aproximadamente, una cuarta parte. La cuota de España en el comercio mundial varía en función de la unidad de medida. Así, si observamos la cuota en metros cuadrados, Italia ocupa el primer lugar con el 33\% del total comercio mundial, seguida de España con un 26,9\%. Sin embargo, si nos fijamos en el porcentaje sobre el valor en euros, Italia detenta casi la mitad del total comercio mundial, $46,8 \%$, frente al $24,9 \%$ de España apreciando, además, la diferencia significativa que existe entre el precio medio de exportación de Italia de $8,84 € / \mathrm{m}^{2}$ y el de España de 5,78 $€ / \mathrm{m}^{2}$. Por lo tanto, observamos, y este es el punto crítico, que Italia consigue unos precios que están en los segmentos más altos de todos los mercados, porque cuando se comparan cifras por valor, en vez de por volumen, Italia rebasa todas las expectativas. Asimismo, si tenemos en cuenta la evolución de los precios, se desprende una tendencia alcista para los precios de Italia, que pasan de $8,62 € / \mathrm{m}^{2}$ en el 2001 a $8,84 € / \mathrm{m} .{ }^{2}$ en el 2002, mientras que, contrariamente, España pasa de 5,86€/ m. ${ }^{2}$ de precio medio en el 2001 a 5,78 $€ / \mathrm{m}^{2}$ en el 2002 (3).

Al mismo tiempo, la mayor presencia de la competencia internacional destaca en el sentido de que España pasa de una cuota de comercio mundial en el 2001 de $28,7 \%$ con $338.967 \mathrm{~m}^{2}$ a una cuota en 2002 de $26,9 \%$ con $356.484 \mathrm{~m}^{2}$, es decir, incluso subiendo en volumen de metros, se observa una bajada en la cuota de participación mundial relativa.

Por otra parte, en Italia la lectura es diferente. Se pasa de una cuota mundial en volumen en 2001 del 37,4\% con $440.749 \mathrm{~m}^{2}$ a un $33 \%$ con $437.698 \mathrm{~m}^{2}$ en 2002 . Es decir, pierde cuatro puntos porcentuales de cuota relativa por la bajada del volumen de sus exportaciones. Análogamente, pasa de una cuota mundial en valor del $49,9 \%$ con 3.798 .250 miles de euros en 2001 a una cuota del 46,8\% con 3.868.971 miles de euros en 2002. Al igual que en el caso de España, la mayor subida relativa de los competidores hace que, en global, se pierda cuota relativa en el ámbito mundial.

Por lo tanto, la diferencia fundamental estriba en que Italia pierde metros cuadrados de exportación y también de producción, pero aumenta precios y valor, mientras que España aumenta metros pero pierde precio, si bien consigue aumentar en valor. No obstante, la mayor competencia internacional hace que pierdan ligeramente peso relativo a nivel mundial.

En consecuencia, observamos como España se sitúa en una situación preocupante. Mientras que Italia aumenta sus precios, aun bajando su venta exterior, y se posiciona en los segmentos más altos del mercado, España, por el contrario, tiende a sufrir contracciones de precios en los diferentes mercados, quedando en una posición intermedia entre Italia y los nuevos competidores internacionales como 
Brasil, Turquía o China. Esto conduce al sector español una posición intermedia nada cómoda. En precios bajos no puede competir, pero tampoco en los altos. Sólo queda la adopción de movimientos estratégicos para intentar posicionarse en los segmentos altos, porque si continua bajando los precios, llegará a competir con los nuevos competidores, lo que resulta complejo en precios.

\section{LA INDUSTRIA CERÁMICA EUROPEA EN EL CONTEXTO GLOBALIZADO}

En el "cluster" de Castellón son variados los agentes que componen los diversos sectores de la cadena de valor sectorial y se localizan en el mismo territorio: proveedores de materia prima, proveedores de esmaltes y fritas, proveedores de maquinaria, empresas de transporte, clientes, distribuidores $\mathrm{y}$ otros sectores auxiliares. Evidentemente, la presencia y fortaleza de los sectores implicados varía. Así, por ejemplo, el sector de esmaltes es líder a nivel mundial, mientras que el de maquinaria está totalmente dominado por los fabricantes de equipo italianos (11), si bien su presencia en el territorio es significativa. Asimismo, se ubican diferentes instituciones públicas que pertenecen a la Red de Institutos Tecnológicos de la Comunidad Valenciana, como el Instituto de Tecnología Cerámica, ITC, y la Asociación para la Promoción del Diseño Cerámico, ALICER, así como otras instituciones y eventos (12).

En la comparación de los "clusters" de Castellón y la Emilia Romagna, se observan claras diferencias en las configuraciones de las cadenas de valor articuladas en dichas localizaciones o clusters, como efecto del paradigma imperante en cada cluster y de las estrategias competitivas seguidas por las empresas localizadas. En general, las empresas italianas son más proactivas en los temas de mercadotecnia y comercialización, al contrario de las españolas que suelen estar más enfocadas en la producción. En este sentido, se aprecia un mayor esfuerzo tendente a buscar la diferenciación como ventaja competitiva. Esto se aprecia, fundamentalmente, en los niveles de precios que obtienen ambos países en los mercados de exportación. Por ejemplo, en EE.UU el precio medio italiano es de 12,26 euros, mientras que el español es de 6,68 euros (3). Dichas configuraciones son el resultado del paradigma imperante en cada cluster y de las estrategias competitivas seguidas por las empresas localizadas.

En el inicio de la cadena de valor, en el proceso de compra de arcilla, atomización y preparación de material, en el distrito de Castellón se articula el proceso de forma más desintegrada estando, por lo general el proceso de atomización de la arcilla separado del fabricante pero presente en el territorio, con lo que se consigue una mayor eficiencia, hecho que unido a la presencia de arcillas en la zona, proporciona en global unas ventajas de coste (13). En la zona de la Emilia Romagna la arcilla se importa de Turquía, Alemania y Ucrania, y el proceso de atomizado se suele realizar por el fabricante, lo que integra más el proceso pero produce mayores costes en global. En la fase del diseño, normalmente, en Castellón se obtiene dicho servicio gratis de las empresas productoras de esmaltes y colorificios, que tienen departamentos propios de diseño mayores que cualquier productora de azulejos, mientras que en Italia la mayoría de las empresas tienen departamento propio de diseño o acuden a estudios del exterior.

En cuanto al producto realizado, en Italia suele realizarse la producción en un 55\%, en gres porcelánico, más dependiente del sector de maquinaria, mientras que en España se realiza en mayor proporción azulejo convencional, más dependiente del proceso de esmaltado, ascendiendo el gres porcelánico a la cifra del $8,5 \%$ de la producción. Además, Italia fabrica en mayor proporción pavimentos que revestimientos. Estos hechos son relevantes, en el sentido de que China está tendiendo, mayoritariamente hacia la producción de gres porcelánico (2), lo que obligará al "cluster" de Castellón a seguir reforzando el azulejo de pasta roja.

El diferente diseño, unido a la diferente tipología de producto ya sirve de base para tener un producto de mayor valor en el caso italiano. Por otra parte, después del proceso de empaquetado y almacenaje, la fase logística, que en el caso español se apoya en mejores infraestructuras viarias que en Italia. No obstante, las empresas italianas han dado un paso adelante con la construcción de la plataforma logística Assocargo, para optimizar la entrada de arcillas y salida de productos finales, si bien en el "cluster" de Castellón existe un proceso de evaluación para la replicación de un sistema similar. A continuación, viene el proceso crítico: la comercialización. La mayoría de los fabricantes cerámicos de Castellón, vía almacenistas en el mercado nacional o vía redes de distribución comercial (representantes) externas, se desentienden de la venta directa, siendo muy pocas las empresas que tienen redes propias de comercialización y aun en menor número tiendas propias. El hecho de no controlar la red de distribución ni la venta final origina que, en ocasiones, los productos estén mal expuestos, mal etiquetados, no se enfatice sus características técnicas y carezcan de marcas comerciales reconocidas. En el caso italiano, suelen tener redes propias de distribución, se suelen disponer de varias marcas incluso para el mismo producto y existe, en definitiva, un esfuerzo intensivo de mejorar y potenciar la parte comercial. En la última feria de CERSAI exponían sus productos 80 empresas dedicadas exclusivamente a la comercialización.

Así, observamos como en las últimas fases de la cadena de valor, exceptuando la colocación, existe una diferencia fundamental. La cadena del valor del distrito de Castellón carece del eslabón de la comercialización, en general. En otras palabras, lo puede tener pero está poco desarrollado, y sólo algunas empresas líderes son conscientes de la necesidad de dichas fase. Esta es, junto con el diseño, la principal diferencia existente entre los dos "clusters".

Además, dicho eslabón de comercialización puede ser la clave del futuro porque puede marcar el posicionamiento de las empresas. Si la distribución sigue la misma tendencia que en el resto de sectores, como el mueble o la alimentación, por ejemplo, el acceso a canales de distribución puede ser la clave de la supervivencia futura. El segmento por el que compiten las empresas productoras de azulejos de Castellón está mas cerca del segmento al que se dirigen las productoras chinas, turcas o brasileñas que al que se dirigen las italianas, con carácter general, de ahí que a largo plazo sea clave la comercialización.

En consecuencia, se pueden extraer varias conclusiones. En Italia se logra una estrategia competitiva más tendente hacia la diferenciación, mientras que en España, se tiende hacía los costes, para una determinada combinación de calidad-precio-segmento, en general. Los eslabones diferentes de la cadena de valor son el gres porcelánico (que es el que más valor añadido produce y mayor margen de diferenciación presenta), el diseño y la comercialización.

En la tabla 1 se apuntan las principales diferencias en lo que respecta a instituciones territoriales en los respectivos 
TABLA I. COMPARACIÓN PRINCIPALES INSTITUCIONES EN LOS ClUSTERS ITALIANO Y ESPAÑOl. COMPARATIVA DE LOS “CLUSTERS” ESPAÑOL E ITALIANO. (2) (27) (28)

\begin{tabular}{|c|c|c|}
\hline & España [Castellón] & Italia [Emilia-Romagna] \\
\hline \multicolumn{3}{|c|}{ CARACTERÍSTICAS DIFERENCIADORAS } \\
\hline Paradigma del cluster & Enfocados a la producción & Volcado hacia la comercialización, vía el diseño, imagen y estilo \\
\hline Ventajas competitivas & $\begin{array}{l}\text { Excelente relación calidad/precio } \\
\text { Presencia de arcillas en la zona } \\
\text { Optimización del proceso productivo } \\
\text { Efectividad de las instituciones } \\
\text { Conocimiento tácito importante } \\
\text { Eficiencia colectiva } \\
\text { Arraigo en la zona (capital social) } \\
\text { Esmalteras líderes nivel mundial, con } \\
\text { inversiones directas internacionales }\end{array}$ & $\begin{array}{l}\text { Diseño } \\
\text { Imagen de marca vía "Made in Italy" } \\
\text { Competencia y enfoque hacia la comercialización (representantes, servicio al } \\
\text { cliente, entrega rápida) } \\
\text { Maquinaria líder nivel mundial, con inversiones directas internacionales } \\
\text { Conocimiento tácito trabajadores }\end{array}$ \\
\hline Debilidades & Poco enfoque a la comercialización & Infraestructuras viarias y de instituciones; débil acción colectiva \\
\hline Estrategias competitivas & $\begin{array}{l}\text { Competencia en gestión del proceso, } \\
\text { liderazgo en costes genérico }\end{array}$ & $\begin{array}{l}\text { Énfasis en diseño } \\
\text { Integración hacia delante } \\
\text { Internacionalizacion } \\
\text { Concentración y diversificación de marcas } \\
\text { Búsqueda de diferenciación }\end{array}$ \\
\hline Integración vertical & Escasa & Media-alta \\
\hline Tejido productivo & Familiar, pymes. Menor tamaño & Grandes grupos y empresas familiares. Mayor tamaño \\
\hline Cadena de valor & $\begin{array}{l}\text { Maquinaria italiana, esmalteros y fabricantes } \\
\text { locales, acción de instituciones importante }\end{array}$ & $\begin{array}{l}\text { Maquinaria local, esmalteros españoles y fabricantes locales, representantes } \\
\text { de ventas, débil acción instituciones }\end{array}$ \\
\hline Estructura comercialización & $\begin{array}{l}\text { Sólo líderes aquellos que poseen redes de } \\
\text { distribución propias, intento de creación } \\
\text { marcas }\end{array}$ & Redes de distribución propias y servicios post-venta \\
\hline Poder en el distrito & $\begin{array}{l}\text { Relaciones no jerárquicas, fuerte posición } \\
\text { esmalteras }\end{array}$ & Fuerte posición de los fabricantes, pero relaciones no jerárquicas \\
\hline Instituciones & Fuertes & Medias \\
\hline Tendencias de producto & $\begin{array}{l}\text { Pasta roja y con tendencia hacia el } \\
\text { pavimento. Poco porcelánico }(8,5 \%)\end{array}$ & Pasta blanca. Hacia la producción total de porcelánico $(55 \%)$ \\
\hline $\begin{array}{l}\text { Tendencia en la estructura } \\
\text { empresarial }\end{array}$ & $\begin{array}{l}\text { No concentración ni fusiones. Excesivo } \\
\text { orgullo de "empresa propia" }\end{array}$ & Concentración y aumento de tamaño \\
\hline Diseño & Bajo, salvo algunos líderes & Medio-Alto \\
\hline Marketing & Bajo, salvo algunos líderes & Medio-alto \\
\hline Ciclo de vida del cluster & $\begin{array}{l}\text { Fase de crecimiento, si bien ya se observan } \\
\text { señales de madurez y tendencia a la bajada } \\
\text { ligera de precios }\end{array}$ & $\begin{array}{l}\text { Madurez, disminución de la producción de un }(-5,16 \%) \text {, pero aumento de } \\
\text { valor y precio. }\end{array}$ \\
\hline Precios y segmentos destino, $€ / \mathrm{m}^{2}$ & Medios y bajos, precio medio 5,33 & Medio-altos y altos, precio medio 8,7 \\
\hline Rentabilidad sobre ventas & $10,4 \%$ & $5,1 \%$ \\
\hline $\begin{array}{l}\text { Esfuerzo inversor } \\
\text { (inversión entre ventas) }\end{array}$ & 0,023 & 0,0504 \\
\hline $\begin{array}{l}\text { Productividad } \\
\text { (valor producción entre inversión) }\end{array}$ & 43,4 & 19,23 \\
\hline \multicolumn{3}{|l|}{ CONDICIONES FACTORES } \\
\hline Asociaciones patronales & $\begin{array}{l}\text { ASCER (fabricantes azulejos), ANNFFEC } \\
\text { (esmaltes) y ASEBEC (maquinaria) }\end{array}$ & ASSOPIASTRELLE (fabricantes azulejos), y ACIMAC (maquinaria) \\
\hline Centros de Formación específica & $\begin{array}{l}\text {-Universitat Jaime I } \\
\text {-Instituto de Promoción Cerámica } \\
\text {-Escuela de artes y oficios de Castellón } \\
\text {-Instituto Caminás de Formación Vocacional }\end{array}$ & $\begin{array}{l}\text { - Universidad de Bolonia y Modena } \\
\text { - Escuela de Arte de Modena } \\
\text { - Cerform }\end{array}$ \\
\hline Centros de investigación & $\begin{array}{l}\text {-Instituto de Tecnología Cerámica } \\
\text {-Asociación para la Promoción y el Diseño } \\
\text { Cerámico } \\
\text { Laboratorio Cerámico Sebastián Carpi } \\
\text {-Instituto Cerámica y Vidrio }\end{array}$ & - Centro Cerámico de Bolonia \\
\hline
\end{tabular}




\begin{tabular}{|c|c|c|}
\hline $\begin{array}{l}\text { Eventos de promoción } \\
\text { internacional }\end{array}$ & $\begin{array}{l}\text {-Feria de Cevisama (azulejos) } \\
\text {-Qualicer (calidad en el proceso cerámico) }\end{array}$ & -Feria de Cersaie (azulejos) \\
\hline Otras instituciones & $\begin{array}{l}\text { Cámaras de Comercio } \\
\text {-Asociación de Técnicos Cerámicos } \\
\text {-Colegio Ingenieros Industriales } \\
\text { - Qualicer }\end{array}$ & -Assocargo \\
\hline Publicaciones específicas & $\begin{array}{l}\text {-Azulejo } \\
\text {-Técnica Cerámica, etc. }\end{array}$ & -Ceramic World Review \\
\hline $\begin{array}{l}\text { Empleo directo fabricantes de } \\
\text { azulejos }\end{array}$ & 26.100 trabajadores directos & 30.749 trabajadores directos \\
\hline \multicolumn{3}{|l|}{ CONDICIONES DEMANDA } \\
\hline Consumo total interior & 327 millones $\mathrm{m}^{2},\left[2^{\circ}\right.$ nivel mundial $]$ & 185 millones $\mathrm{m}^{2}, 5^{\circ}$ nivel mundial $]$ \\
\hline Consumo per cápita interior & $8.2 \mathrm{~m} 2 /$ habitante, $\left[1^{\circ}\right.$ nivel mundial $]$ & $3,2 \mathrm{~m} 2 /$ habitante, $\left[4^{\circ}\right.$ nivel mundial $]$ \\
\hline \multicolumn{3}{|l|}{ INDUSTRIA AUXILIAR } \\
\hline $\begin{array}{l}\text { Empresas esmalteras. Facturación } \\
\text { esmalteras y trabajadores }\end{array}$ & $\begin{array}{l}26 \text { empresas } \\
835,9 \text { millones euros } \\
3487\end{array}$ & \\
\hline $\begin{array}{l}\text { Empresas maquinaria cerámica y } \\
\text { trabajadores. Facturación }\end{array}$ & $\begin{array}{l}41 \\
700 \text { empleos directos }\end{array}$ & $\begin{array}{l}171 \text { empresas } \\
1425 \text { millones euros } \\
6500 \text { empleos directos }\end{array}$ \\
\hline \multicolumn{3}{|l|}{ ESTRUCTURA } \\
\hline $\begin{array}{l}\text { Empresas de azulejos } \\
(27)(28)\end{array}$ & $\begin{array}{l}265 \\
\text { Estructura media mayoritaria en el rango de } \\
\text { hasta } 100 \text { trabajadores. Predomina } 25-50 \text {. } \\
\text { El } 8 \% \text { de la industria acumula el } 38,6 \% \text { de la } \\
\text { producción } \\
\text { La empresa que más produce constituye el } \\
3,6 \% \text { de la producción total en } \mathrm{m}^{2} \text {. }\end{array}$ & $\begin{array}{l}241 \\
\text { Estructura principal en el rango de } 100 \text { a } 200 \text { trabajadores. } \\
10 \text { grupos concentran el } 61,5 \% \text { de la producción. }\end{array}$ \\
\hline \multicolumn{3}{|l|}{ POSICIONAMIENTO } \\
\hline Posición en mercado americano & $\begin{array}{l}\text { Seguidores en el mercado de EE.UU. } \\
\text { Segmentos medios y bajos } \\
\text { Líder en América Latina }\end{array}$ & Líderes en EE.UU, segmentos medio-altos y altos \\
\hline Posición en mercado Europeo & $\begin{array}{l}\text { Líder en Portugal y UK e Irlanda. En el resto } \\
\text { de Europa Occidental, seguidores. } \\
\text { En UK España situada en segmento medio y } \\
\text { medio alto, pero no en el alto. }\end{array}$ & $\begin{array}{l}\text { Líder en Alemania, Francia, Austria, Bélgica y Luxemburgo, Dinamarca, } \\
\text { Finlandia, Grecia y Países Bajos, Suiza y Noruega }\end{array}$ \\
\hline $\begin{array}{l}\text { Posición en Mercado de Europa } \\
\text { del Este }\end{array}$ & $\begin{array}{l}\text { Líder en Rusia, Bulgaria, Rumanía, Ucrania y } \\
\text { Lituania. El segmento alto se lo lleva Italia }\end{array}$ & Líderes en Croacia, Eslovaquia, Eslovenia, Hungría y Polonia \\
\hline \multicolumn{3}{|l|}{ PRODUCCIÓN Y EXPORTACIONES } \\
\hline Producción, millones de $\mathrm{m}^{2}$ & 651 & 605 \\
\hline Valor de la producción mill. $€$ & $3.448,03$ & $5.318,62$ \\
\hline Exportación millones de $\mathrm{m}^{2}$ & 357,15 & 437,6 \\
\hline Precio medio exportación, $€$ & 5,77 & 8,84 \\
\hline Cuota producción mundial en $\mathrm{m}^{2}$ & $10,5 \%,[+2 \%]$ & $9,8 \%,[-5,2 \%]$ \\
\hline Inversiones miles euros & 91.140 & 26.8163 \\
\hline $\begin{array}{l}\text { Productividad en } \mathrm{m}^{2} / \\
\text { trabajadores(2) }\end{array}$ & 0,0249 & 0,0196 \\
\hline \% gres porcelánico / producción & 8,5 & 54,37 \\
\hline $\begin{array}{l}\% \text { Pavimentos entre producción } \\
\text { total }\end{array}$ & 63,0 & 79,8 \\
\hline
\end{tabular}


"clusters".

En líneas generales, se observan pocas diferencias en cuanto a las dotaciones institucionales en ambos clusters, estando ambos dotados de suficientes mecanismos públicos y privados para realizar una buena labor de apoyo a la cadena de valor, si bien parece ser que la interacción y ciertas instituciones como el ITC o el ICV funcionan mejor en el caso de Castellón (2); (14), (5). Además, la concentración y al aumento de tamaño de las empresas italianas propicia el que se pierda acción colectiva. Asimismo, también se observa, el diferente enfoque existente entre la orientación química en Castellón por la fortaleza del sector de fritas y esmaltes con el desarrollo de una titulación de Ingeniería Química Cerámica en la UJI (12) que parte de la iniciativa del ITC (16) y el de maquinaria en Italia con las titulaciones de Ingeniería Mecánica Cerámica en Ancona y Bolonia, la asociación ACIMAC, etc.

\section{LA DEMANDA TECNOLÓGICA EN EL SECTOR CERÁMICO. EL PROYECTO MONOTONE.}

El proyecto MONOTONE se plantea como objetivo la modelización de la línea de producción de decoración y su futura automatización. Como principal motivacion se puede considerar la mejora de la calidad del producto y su sostenibilidad, su repetibilidad, etc. Se pretende posibilitar una producción justo a tiempo de modo que se pueda trabajar contra pedido y poder producir series cortas con un cambio de producto rápido, mejorar el nivel de calidad y reducir el nivel de inventario y los stocks. El mismo está liderado por la Universidad Politecnica de Valencia y colaboran como socios 15 empresas españolas, italianas, del Reino Unido, Suecia y Austria, todas ellas del sector cerámico.

Dentro de este proyecto se ha planteado la necesidad de conocer la demanda tecnológica potencial de la línea de decoración ${ }^{2}$ frente a los retos futuros del sector cerámico. Para ello, se entrevistó a más de 40 profesionales del sector en España, Italia y Reino Unido y se mantuvieron dos mesas redondas en Castellón (17) y Módena (18). En los párrafos siguientes trataremos de resumir las conclusiones de las mismas.

Se preguntó a los entrevistados sobre las aplicaciones más comunes en la línea de decoración y las repuestas indican un predominio de determinadas operaciones y cierta penetración de métodos avanzados como la flexografía, el control automtizado de calidad y solo una presencia testimonial de máquinas digitales como el sistema kerajet ${ }^{\circledR}$.

Las respuestas relativas al método de trabajo en los pedidos, indican que en un $80 \%$ de los casos se trabajaba contra stock y solo en un $20 \%$ contra pedido. Un pequeño porcentaje 3,2 \%trabajaba en un $90-95 \%$ contra pedidos del cliente Estas, a pesar de ser de tamaño pequeño, habían adquirido e implantado dos unidades de impresión digital kerajet ${ }^{\circledR}$ y trabajaban casi con filosofía justo a tiempo.

Como ya se ha apuntado, la variedad de modelos con los que se trabaja en las empresas es enorme lo que complica el proceso logístico de control de inventario.

Con relación a la facilidad de incorporación de nuevas tecnologías a la línea de producción, la respuesta era positiva en un $70 \%$ de las empresas. No obstante, la conclusión indica que únicamente las empresas grandes se han organizado para absorber la tecnología de una manera sistematizada. Este factor es crítico y está ligado a la cultura artesanal existente en el proceso de decoración.
Con relación a los controles de las desviaciones en los parámetros de producción, en el $85 \%$ de los casos se llevan a cabo con mayor o menor frecuencia, pero solo un $30 \%$ de los respondientes afirmaban que los datos técnicos de control se conservaban y analizaban. Esto refleja la cultura artesanal que aún domina la industria. Por el contrario, las respuestas de las empresas del Reino Unido e Italia reflejan una cultura taylorista en los procedimientos y métodos, el control etc. Las empresas más grandes, y especialmente aquéllas que han implantado ya sistemas de gestión, archivan información y datos sobre el rendimiento de la producción y las variables técnicas. Este hecho será una barrera a la modelización de la producción, no sólo debido a la escasez de datos, sino también por ser un reflejo de una cultura reacia a los controles de producción, excepto cuando estos se incorporan a los equipos, como en el caso de hornos, prensas, equipos de logística, etc.

Las empresas más grandes tienen procedimientos de control de producción y toma de muestras, con frecuencia horaria en algunos casos. A las mismas conclusiones llega un estudio más exhaustivo recientemente publicado (19).

Cuando se inquiere sobre el control del nivel de Calidad, en general las respuestas reflejan una preocupación importante en este aspecto. En el segmento más alto de la muestra, básicamente las empresas más grandes, se apunta un nivel de calidad del $95-96 \%$ con el $20 \%$ de las respuestas. En el segmento siguiente, este nivel es del 90 - 95\% con el $60 \%$ de las respuestas. Otros respondientes apuntan un rango del 86 $90 \%$. La cultura de las segundas calidades parece estar en vías de extinción, aunque los respondientes reconocen que algunas empresas distribuyen este tipo de segmento de producto. En general, el nivel de calidad resulta una pregunta difícil debido a las normas del sector y el control humano final de la línea de producción, que supone dudas sobre su consistencia. Cuatro empresas comercializan máquinas de clasificación automática, aunque su eficacia generalmente se cuestiona y parece que el control humano de calidad es aún necesario. En cualquier caso, llamamos la atención del lector sobre lo comentado más arriba respecto a la segmentación de la demanda del producto. Un $95 \%$ de las respuestas confirmaban el aumento en los objetivos de calidad, año tras año. La mejora continua parece ser una filosofía que gana adeptos en la industria cerámica.

Con relación al efecto de los cambios en la línea de decoración y la estabilización posterior de la línea, en general, los cambios de diseño de producto llevaban de 0,5 a 2 hrs. como promedio. Otros respondientes, que trabajaban con series más cortas apuntaban períodos más cortos (10 -30 minutos) debido a sus habilidades y entrenamiento. En general, se reconoce que la estabilización de la línea es un problema más complejo y puede suponer 4 - $5 \mathrm{hrs}$. Debe tenerse en cuenta que el único medio de comprobación es observar el producto tras su paso por el horno ${ }^{3}$. El cambio de tamaño se reconoce, en general, como el problema más difícil y el que requiere más tiempo para resolver. El promedio de cambio de tamaño supone un período del orden de 1 a $4 \mathrm{hrs}$. y un periodo más grande para la estabilización de la línea con 2-4 hrs.

Los usuarios de la tecnología kerajet ${ }^{\circledR}$, decoración por inyección de tinta, apuntan que, con este sistema, los cambios de pedido de tamaño o modelo pueden llevarse a cabo en un promedio de $0,5 \mathrm{hrs}$. y la línea estabilizarse en $1 \mathrm{hr}$. como media.

Con relación a la complejidad de las operaciones en la línea de producción, las respuestas coinciden: en primer lugar aparece el cambio de tamaño con un $90 \%$ de repuestas, en segundo lugar la estabilización de la línea, y en tercer 
lugar las operaciones de decoración, especialmente las referidas al sistema Rotocolor $^{\circledast}$ que se reconocía por todos los respondientes, con algunas excepciones, como una operación muy inestable.

Como consecuencia de lo anterior, existe un exceso de líneas de decoración respecto a las de cocción del orden de 30 a $100 \%$ lo que permite el cambio y preparación de pedidos con cierta flexibilidad.

En general, los respondientes coinciden en que hay una tendencia clara en la reducción del tamaño de las series. Hoy la serie de $800 \mathrm{~m}^{2}$ no es rara, aunque el rango está entre 2.000 y $10.000 \mathrm{~m}^{2}$. En general, las líneas trabajan con tres cambios de turno durante la semana. El $40 \%$ de las empresas mantienen los hornos inactivos durante el fin de semana, no obstante esta situación depende de la demanda.

Cuando se pregunta sobre los procedimientos de operación, un $15 \%$ de los respondientes han sido certificados con ISO 9000, lo que coincide con las estadísticas disponibles sobre el mercado español. El porcentaje para el italiano y otras empresas europeas es bastante mayor.

Las empresas líderes están incorporando, o ya han incorporado, sistemas de gestión integrados, del tipo ERP, como SAP R3, DPM, etc. que incluyen la gestión de los datos de producción. Este hecho favorecerá el futuro de la gestión de datos de la producción y la implantación de una filosofía de gestión de la producción que requerirá su modelización en el futuro (19).

Como, se ha apuntado, los objetivos del proyecto MONOTONE son automatizar la línea de decoración por medio del modelado del proceso, la instalación de sensores y la realimentación de sistemas de control, siguiendo la tendencia de la industria manufacturera. En este sentido, sólo un $10 \%$ de los respondientes los encontraba alcanzables y un $90 \%$ deseables. Un $80 \%$ de respuestas los encontraba utópicos.

En cuanto a las barreras fundamentales para alcanzar estas metas, las respuestas indicaban su importancia, valoradas de 5 a 1, de mayor a menor: exceso de variables de proceso 4,5; falta de especificaciones y estandarización en esmaltes y pigmentos, 3,9; bajos niveles de formación en los empleados, 3,5; conservadurismo del personal, 3, 4; falta de procedimientos, 3,2; falta de conocimiento sobre el proceso, 2; y problemas técnicos en los equipos, 1,8 .

En general puede concluirse que nos enfrentamos con un proceso con un número excesivo de variables, siendo unas dependientes de otras en un sistema complejo. La ausencia de estandarización de esmaltes supone una dificultad añadida. El enfoque artesanal del proceso, por parte del personal, implica que la incorporación de la tecnología al mismo resulte muy compleja. Esto puede ser una de las causas de los problemas sufridos por un sistema digitalizado como kerajet dónde su difusión esta encontrando ciertas dificultades por parte del personal de producción (20). Ésta es una cuestión que se suscitó también en la mesa redonda que se llevo a cabo en ACIMAC y fue apuntada por los fabricantes italianos de maquinaria, y requerirá esfuerzos especiales en el proceso de difusión de nuevas tecnologías (18).

Un caso especial son las empresas de tercer fuego de piezas especiales. Sus respuestas reflejan un enfoque diferente al resto del sector. Estas empresas trabajan básicamente para otros fabricantes que diseñan sus modelos para encajar en los catálogos de sus clientes. Sus respuestas reflejan un acercamiento aún más artesanal donde el rol de la tecnología es limitado

Las tres mesas redondas citadas han confirmado los resultados de la encuesta. Confirman los aspectos artesanales de la operación en la línea de decoración. Algunas empresas italianas argumentan que sus clientes no aceptarían productos tan estandarizados como los que se obtendrían con las tecnologías propuestas. También los fabricantes de esmaltes y pigmentos, argumentan por su parte, la dificultad de transferir sus desarrollos de laboratorio en el diseño de modelos a la línea de producción por estar basados en conocimiento tácito no explicitado. Se acepta que el modelo productivo, basado en grandes series, ya no tiene futuro y también existe coincidencia en la necesidad de reducir el inventario que han crecido, en Italia y España a niveles difíciles de financiar. Se asocia, en el sector de maquinaria, la automatización con aumento de los precios de los equipos que los clientes no están dispuestos a asumir. Por su parte, los fabricantes italianos opinan que no es factible automatizar la producción de un producto tan sofisticado. También se apunta a la necesidad de un desarrollo de producto concurrente ya que a veces diseñadores y técnicos de producción hablan diferentes idiomas.

Un tema recurrente en ambas mesas fue la necesidad de una discusión y un análisis sobre las estrategias de las empresas y su orientación de producto ya que ello influye en las necedades tecnológicas de la empresa.

\section{LAS TENDENCIAS TECNOLÓGICAS EN EL SECTOR CERÁMICO.}

En este último apartado trataremos de analizar cuales son las tendencias y las últimas innovaciones, que en la dirección de la automatización han aparecido y se han implantado en el sector.

Podría decirse que el éxito comercial de la tecnología Rotocolor $^{\oplus}$ presentada en 1995, y de la que existen hoy más de 5.000 unidades instaladas, supone un hito en la industria cerámica. Su patente significó la introducción de un sistema revolucionario aceptado en la industria del azulejo como una nueva norma en el proceso de decoración (21). La posibilidad de usar rodillos especiales extiende sus funcionalidades para decorar pavimentos, permitiendo decorar superficies muy irregulares y aplicar diversas posibilidades de decoración. También ofrece el potencial de una calidad de tono constante, una velocidad productiva más alta, minimiza los desechos en la línea de esmaltado, etc.

Un caso de éxito es el del horno. Tras el desarrollo de los hornos de monococción, los hornos han sofisticado su diseño y han incorporado mejoras continuas incluyendo la adaptación para gas, el ahorro de energía, y recientemente la ingeniería de control. Mención especial debe hacerse del proyecto europeo BRITE EURAM BE 573, AXIOM, que ha desarrollado, entre otras la modelización del proceso de cocción. La feria de Technargilla de 2003 exhibía un software de gestión de proceso basado en los resultados del proyecto, siendo el único sistema ERP mostrado en la feria.

Otra área que ha experimentado importantes desarrollos ha sido la del control y clasificación automática de la calidad. Varios fabricantes tienen productos en el mercado: Cerascreen de Massen, El Scanmaster ${ }^{\circledR}$ de Italvision, y el Select Master ${ }^{\circledR}$ de Surface Inspection, empresa pionera en este área y que abrió el mercado en 1999. Los fabricantes de estos sistemas argumentan una mejora en las normas de calidad, una reducción de los costes operacionales y un criterio claro y constante de selección y uniformidad. Existe cierta controversia sobre la eficacia de estos sistemas, ya que cada fabricante defiende las excelencias 
de sus sistemas mientras los clientes, los fabricantes del azulejo, expresan sus dudas y todavía confían en la clasificación manual en sus rutinas diarias. Parece que estos sistemas tienen problemas con ciertos diseños como los azulejos rústicos. Un fabricante de estos equipos - Massen - arguye que su sistema Cerascreen, puede incluso controlar la calidad de las baldosas antes del horno, aunque esto no se ha demostrad en ninguna publicación. Este fabricante ha publicado referencias de una comparación entre la clasificación manual y la automática, mostrando ventajas de este sistema (22)

Con relación al proceso de esmaltado, un sistema avanzado relevante es el de Eurotecnica, el sistema Vela. Este se basa en una tecnología de esmaltado innovadora desarrollada por Ingegneria Ceramica. Permite una calidad en la aplicación de esmaltes muy elevada. Éste es uno de los ejemplos más recientes de automatización en la línea de esmaltado. La demanda de tecnología latente de estos sistemas se demuestra por el éxito de este producto a pesar de su precio, mucho más elevado que los sistemas convencionales (23).

Desde el sector español, se ha desarrollado una línea de esmaltado integrada. Según sus diseñadores presenta varias ventajas técnicas: Permite imprimir informes, almacenar datos de producción, alarmas, etc. lo que permite cambios en los parámetros de la máquina; etc. En la misma dirección se ha presentado un sistema en Technargilla de una empresa italiana. No obstante, estos sistemas se centran, sobre todo, en variables mecánicas.

Se han desarrollado esfuerzos en el dosificado de esmaltado para controlar y supervisar la preparación y dosificación de esmaltes (24).

La utilización de tecnología láser que permite la decoración del azulejo con un proceso de digitalización ha contribuido a la automatización de la línea de decoración. Se utiliza en la producción de pavimento y el grabado de rodillos para la impresión de baldosas, como atestigua uno de los últimos Premios ALFA de CEVISAMA.

Las tecnologías de Información y Telecomunicaciones, T.I., se van incorporando lentamente a la línea de proceso cerámica. Hasta ahora, como ya se ha comentado, lo han hecho en prensa y cocción pero no en la línea de decoración. Nuevas empresas de origen T.I. están incorporándose al sector de maquinaria y líderes clásicos como System han desarrollado su propia división de electrónica. No obstante, se requieren actitudes más innovadoras por parte de los adoptadores para facilitar su incorporación al proceso ya que este es, de momento, un proceso de empuje más que de demanda tecnológica (25).

Los cambios futuros en las fases últimas de la cadena de valor, fundamentalmente en la distribución y su conexión con la fabricación, van a incidir en la demanda tecnológica dirigiéndose hacia la fabricación flexible como ha ocurrido en otros sectores.

\section{CONCLUSIONES}

En la comparación de los clusters de Castellón y la Emilia Romagna, se observan claras diferencias en las configuraciones de las cadenas de valor articuladas en dichos clusters, como resultado del paradigma imperante en cada cluster y de las estrategias competitivas seguidas por las empresas localizadas en ellos.

Hemos observado como los últimos eslabones de la cadena de valor del cluster de Castellón parece que no se tengan en cuenta, en general, por el sector, como si la cadena de valor estuviera cortada. En otras palabras, las actividades del final de la cadena, en ocasiones están, pero muy poco potenciadas, y sólo algunas empresas líderes son conscientes de la necesidad de dichas fases y las incorporan de forma integral.

Conviene señalar que los eslabones finales de la cadena de valor, todos ellos relacionados con el diseño y la comercialización, pueden ser la clave del futuro porque influyen en el posicionamiento de las empresas. No obstante, el constatar que las empresas sin funciones de marketing o diseño, no han pensado en incorporarlas en el futuro resulta paradigmático, en tanto en cuanto obtenemos evidencia empírica de que la existencia de departamento de mercadotecnica y diseño, ambos, están directamente relacionados con los precios obtenidos, en consecuencia, con el segmento atendido y, en última instancia, el posicionamiento de la empresa. Así, el segmento por el que compiten las empresas productoras de azulejos de Castellón está mas cerca del segmento al que se dirigen los productores emergentes que al que se dirigen los italianos, con carácter general, de ahí que a largo plazo sea clave la comercialización.

El diferente paradigma estratégico observado, en los dos clusters comparados, se traduce en la diferente composición de los mercados internacionales y los precios conseguidos por productores españoles e italianos. Prácticamente en todos los mercados, el segmento de gama medio-alta y alta está copado por los últimos, además en países líderes de consumo como Francia, Alemania o EE.UU.

Optar por la diferenciación es la clave del futuro del sector en Europa, por lo que se debe apostar por las actividades de comercialización en general y diseño, realizándolo todo de forma integrada. La estrategia general seguida por el distrito de Castellón, de forma colectiva ha sido válida con las condiciones que han existido. Sin embargo, las reglas del mercado del sector están cambiando (26).

En consecuencia, el paradigma del azulejo de Castellón, enfocado en la mayor parte a la producción, puede no ser válido en los años venideros. Así, deben acometerse los cambios necesarios. Se debe realizar un esfuerzo en crear una imagen de marca conjunta, algo certificable como la denominación de origen. Sin embargo, debemos preguntarnos si realmente interesará a todas las empresas del distrito, especialmente a aquellas que ya aportan por imagen propia alguna diferenciación. Hace falta, en definitiva, el "made in Spain", como sinónimo de algo que aporta valor, que diferencia y que puede ser, en su caso, factor determinante a la hora de la compra, ya que en el caso de los italianos, el "made in Italy" está muy avanzado. Asimismo, todo ello debería acompañarse de acciones conjuntas encaminadas a racionalizar todos los procesos del sector, promoviendo la cooperación, etc. Es más, sería conveniente debatir la necesidad de realizar grupos, ya sea en forma de cooperación formal (formas jurídicas apropiadas) o informal tendentes a buscar tamaño, planteándose, incluso, la especialización de productos por empresas de un mismo grupo, etc. Existe consenso de que las empresas realizan muchos formatos y diseños cuya vida útil no supera la de la feria, fundamentalmente, porque la mayoría de la empresas ofrece los mismos, en esencia por la dependencia general que existe de la empresa productora de esmaltes y pigmentos.

Por último, hay que potenciar todos los elementos que nos conduzcan a la diferenciación, sobre todo la parte del diseño. La tradicional forma en la que el diseño se obtiene de la proveedora de esmaltes, si bien válida en anteriores años, se vislumbra insuficiente para seguir compitiendo. Las 
relaciones con este deben cambiar.

Como consecuencia de lo anterior se puede prever cierta tendencia a fabricar contra pedidos al agilizarse la cadena inferior de distribución y reducirse el stock. Todo ello debe llevara a un paradigma de producción ligera, como ha ocurrido en otros sectores manufactureros. Además, como ya están haciendo algunas empresas líderes, se producirá una introducción progresiva de las tecnologías de la información en producción y una demanda de control de la producción en el área de decoración con una difusión más extensa de la decoración digital y el control de calidad automático.

\section{BIBLIOGRAFÍA}

1. J. Albors. "Networking and technology transfer in the Spanish Ceramic Tiles Cluster. Its role in the sector competitiveness", Int. Jour. of Technology Transfer, 27(3) 263-273 (2002),

2. J.L. Hervas, J. Albors, J.I. Dalmau, "Governance en la cadena de valor sectorial y asimetrías en la performance. Evidencia empírica de las relaciones de coordinación en un sector industrial", Proceedings, Congreso ACEDE, Murcia (2004)

3. ASCER, Informe Anual, Castellón (2003)

4. M.J. Enright. "Organization and Coordination in Geographically Concentrated Industries", en N.Lamoreaux, D.Raff, D. (Eds.), Coordination and Information: Historical Perspectives on the Organization of Enterprise, Chicago University Press for the NBER. Chicago (1995)

5. S. Brusco. "El concepto de distrito industrial: su génesis." en F. Pyke, G. (9) G. Becattini y W. Sengerberger (Eds.) Los distritos industriales y las pequeñas empresas. Ministerio de trabajo y Seguridad Social. Colección Economía y Sociología. (I) 25-37 (1992).

6. J. Albors, X. Molina. "La difusión de la innovación como factor competitivo en las redes inter organizativas descentralizadas. El caso de la industria cerámica valenciana”, Economía Industrial, III 339167-179 (2001)

7. M. Piore y C. Sabel. "The Second industrial divide", pp 145-192 Basic Books, New York. (1984)

8. G. Becattini. "Mercato e forze locali: il distreto industriale", Il Mulino (1987).

9. F. Pyke, G. Beccattini, y W. Sengenberger "Industrial districts and inter-firm cooperation in Italy", International Institute of Labour studies, Geneva (1990)
10. F. Sforzi.“Importancia cuantitativa de los distritos industriales marshallianos en la economía italiana"en F. Pyke, G. Becattini y W. Sengenberger (Eds.) Los industriales y las pequeñas empresas. Ministerio de Trabajo y Seguridad Social. Colección Economía y Sociología del Trabajo. (1) 5 175-193 (1992).

11. J. Albors. "Estudio diagnóstico sobre el sector de maquinaria para la industria cerámica", Técnica Cerámica, 8-12 Anuario (2000).

12. A. Escardino. "La innovación tecnológica en la industria cerámica de Castellón", Bol. Soc. Esp. Ceram. V., 40, 1, 43-52, (2001)

13. E. Criado, M. Regueiro, E. Sánchez. "La industria cerámica en España (1990-2000)", Bol. Soc. Esp. Ceram. V., 40, 6, 413-428 (2001)

14. J. Meyer-Stamer, S. Seibel, y C. Maggi "Improving upon Nature". Report 54/2001. University of Duisburg. (2001).

15. T. Carpi, J. Such, M. Torrejón. “Competitividad, estrategia empresarial y territorio. El caso de la industria textil Valenciana", Economía Industrial, 308, 139-148 (1996)

16. E. Criado, E. Sanchez, M. Regueiro. “La industria cerámica española, ¿ante un cambio de ciclo?" Bol. Soc. Esp. Ceram. V., 43 [1] 85-101. (2004)

17. C. Romagosa, "El Proyecto Monotone", Técnica Cerámica. 311,. 200-225. (2003)

18. Ceramic World Review, 52, 48-49. (2003)

19. B. Segura, E. Vallada, C. Maroto, R. Ruiz, "Análisis del sistema de operaciones en empresas del sector cerámico español", Bol. Soc. Esp. Ceram. V.,43, 6, 929-932 (2004)

20. C. Romagosa. "Kerajet. perfilando el contorno del abismo" Técnica Cerámica. 310, Págs. 30-40. (2003)

21. M. Russo "Innovation dynamics and industrial dynamics in a local production system", Universita di Modena, (2000)

22. R.Massen, T. Franz, (2001), "Tile ceramic automatic Classification" Intern. Ceram., 2, 75-83 (2001)

23. J. Albors. "Technical Demand Analysis in the Tile Decoration Line", Deliverable 2, Monotone Project, European Commission, Brussels, 2003.

24. M. Vicent, "Sistema de control para la dosificación y trasvase automáticos de esmaltes en la industria cerámica", Técnica Cerámica, 306, 1128-1131 (2002).

25. J. Sastre, Técnica Cerámica, 316, 1034-1040 (2003).

26. T. Bursi. "Industria cerámica italiana ed industria cerámica spagnola: due leadership a confronto", ATC, VI Congreso Int. del Técnico Cerámico, Castellón (2001).

27. KPMG, Informe KPMG sobre el sector azulejero español, Economia 3 (2003)

28. Ceramic Word Review, 50, 23-30, (2003)

Recibido: 03.04 .04

Aceptado: 13.01.05 